\title{
AVALIAÇÃO DE RISCO ERGONÔMICO EM ATIVIDADE NO SETOR DE FLORICULTURA
}

\author{
Jhosefe Bruning ${ }^{*}$, Miguel Chaiben Neto ${ }^{1}$, Silvana Antunes Rodrigues ${ }^{1}$, Bruna Dalcin Pimenta ${ }^{1}$, Airton dos Santos Alonço ${ }^{1}$ \\ Tiago Rodrigo Francetto ${ }^{2}$ \\ ${ }^{1}$ Departamento de Engenharia Rural, Universidade Federal de Santa Maria, 97105-900, Santa Maria, RS, Brasil. \\ ${ }^{2}$ Coordenadoria Acadêmica/UFSM-Campus Cachoeira do Sul, CEP:96506-322.Cachoeira do Sul, RS, Brasil.
}

*E-mail:jhosefe.b@gmail.com

Recebido em: 03/07/2019

Aceito em: 22/10/2019

\section{RESUMO}

Nos últimos anos, o setor de floricultura apresenta elevado crescimento. No entanto, há um problema enfrentado nesse setor, ligado aos cuidados com a saúde dos trabalhadores. Desta forma, o objetivo deste estudo é realizar a análise ergonômica do trabalho (AET), aplicada ao posto de trabalho dos alunos de pós-graduação no setor de floricultura. O estudo foi realizado em um ambiente protegido, no setor de floricultura do Colégio Politécnico da Universidade Federal de Santa Maria. Para realizar a análise ergonômica do trabalho, foram avaliadas as atividades desempenhadas pelos alunos de pós-graduação durante a condução de experimentos científicos. Assim, foram analisadas cinco atividades, a saber: a avaliação de crescimento de plantas, o preenchimento da planilha de dados in loco, a realização de irrigação dos vasos por meio de pesagens, a movimentação dos vasos e a avaliação final das plantas. Para a realização das análises, foram coletados dados para a avaliação postural por meio de observações visuais dos trabalhadores, durante a execução das atividades, além de registros fotográficos e vídeos. Posteriormente, foi realizada a análise detalhada, através da aplicação dos métodos RULA, REBA, com o auxílio do software Ergolândia 7.0 e Diagrama de Corlett. Com isso, a análise ergonômica do trabalho possibilitou a verificação das situações que podem desencadear a dor e o desconforto nos trabalhadores, além de evidenciar que as atividades analisadas não apresentaram conformidade quanto à postura empregada, necessitando de intervenção imediata.

Palavras-chave: Ergonomia. Ambiente protegido. Rula. Reba. Diagrama de Corlett.

\section{Introdução}

O setor de floricultura apresenta elevada taxa de crescimento nos últimos anos, segundo Pessa e Pereira [1]. Entretanto, um problema recorrentemente enfrentado consiste na atenção aos cuidados com a saúde dos trabalhadores, cuja execução de posições, na maior parte da jornada de trabalho, ocasionam a fadiga ou a dor muscular ao trabalhador [2].

Essas posições geram aos trabalhadores diversas lesões, traumas cumulativos, doenças e acidentes de trabalho. Além disso, é atestada a presença de sintomas musculoesqueléticos frequentes, principalmente nos membros superiores. Os movimentos repetitivos e as posturas inadequadas podem, assim, causar dores na região lombar e nos membros inferiores [3-4].

Atualmente, a preocupação com a saúde ocupacional dos trabalhadores, que envolve as relações com a saúde, a segurança e a prevenção de doenças ocupacionais tem recebido a devida importância [5-7].

Um requisito de grande relevância ao sistema produtivo de qualquer setor é a saúde do trabalhador. A busca de melhores condições de trabalho está diretamente ligada com a ergonomia, que tem um papel fundamental dentro das empresas, visto que busca a adaptação do trabalho ao homem através da análise da tarefa, das atividades, das posturas e dos movimentos realizados pelo trabalhador, no intuito de proporcionar uma melhor qualidade de vida e um aumento de produtividade [8]. Por isso, a adoção de posturas inadequadas relacionadas ao trabalho são problemas graves, frequentemente desenvolvidos em trabalhadores de diversos setores [9-10].

Assim, a ergonomia atua de forma a amenizar a fadiga dos trabalhadores e possibilitar melhores condições de trabalho e segurança e, como resultado, há o aumento de produtividade do sistema, que contribui de forma direta na motivação pessoal e 
profissional dos trabalhadores [10]. Dessa forma, o objetivo deste estudo consiste em realizar a análise ergonômica do trabalho (AET), aplicada ao posto de trabalho dos alunos de pós-graduação, no setor de floricultura, durante a condução de experimentos em ambiente protegido, ao avaliar as suas condições de trabalho.

\section{Metodologia}

O presente estudo foi realizado em um ambiente protegido, no setor de floricultura do Colégio Politécnico da Universidade Federal de Santa Maria (UFSM), localizado a 2943'17,2" S 53 43'09,6" W, no município de Santa Maria, RS, em maio do ano de 2019 .

Para a análise ergonômica do trabalho (AET), foram avaliadas as atividades que eram desempenhadas pelos alunos de pós-graduação, durante a realização de seus respectivos experimentos científicos. Com isso, foram analisadas as seguintes atividades desempenhadas, a saber: Avaliação de crescimento de plantas (1), Preenchimento da planilha de dados in loco (2), Realização de irrigação dos vasos por meio de pesagens (3), Movimentação dos vasos (4) e Avaliação final das plantas (5).

Para a realização das avaliações, foram aplicadas as ferramentas de análise biomecânica de RULA (Rapid Upper Limb Assessment), REBA (Rapid Entire Boby Assessment), através do Software Ergolândia 7.0 e aplicado o Diagrama de Corlett, a fim de quantificar as regiões do corpo, mais afetadas pelos distúrbios para determinar o nível de desconforto dos trabalhadores mencionados.

O método RULA consiste na avaliação da exposição dos trabalhadores aos fatores de risco que podem ocasionar transtornos nos membros superiores do corpo, devido à sobrecarga biomecânica, ao avaliar o posicionamento de braço, de antebraço, de punho, da rotação do punho, do pescoço e do tronco, além de considerar o uso da musculatura para o desenvolvimento da atividade executada, bem como a carga necessária na operação. Por fim, cada fator recebe uma determinada pontuação que expressa o nível de intervenção necessária (Tabela 1).

Tabela 1. Tabela 1. Descrição da pontuação para cada nível de intervenção do método RULA

\begin{tabular}{cc}
\hline Categorias & Nível de intervenção \\
\hline 1 & Não são necessárias medidas corretivas \\
2 & São necessárias correções em um futuro próximo \\
3 & São necessárias correções tão logo quanto possível \\
4 & São necessárias correções imediatas \\
\hline
\end{tabular}

Fonte: Adaptado de Ergolândia 7.0.
O método REBA consiste na avaliação da atividade a partir de uma observação dos fatores que causam danos aos executores, em função dos ciclos laborais, tais quais; as posturas do tronco, de pescoço, das pernas, dos braços, dos antebraços e dos punhos, pontuados em tabelas para cada grupo, especificamente. $\mathrm{O}$ método avalia as posições potencialmente prejudiciais ou frequentes [11].

A sua aplicação resulta em uma pontuação final, em que são definidos, por meio de uma tabela, os níveis de risco e a ação de intervenção recomendada (Tabela 2).

Tabela 2. Pontuação para determinar o nível de intervenção ergonômica necessária pelo método REBA

\begin{tabular}{ccc}
\hline Pontuação & Significado & Nível de intervenção \\
\hline 1 & $\begin{array}{c}\text { Risco } \\
\text { insignificante }\end{array}$ & Não é necessária \\
2 ou 3 & Risco baixo & Pode ser necessária \\
4 a 7 & Risco médio & Necessária \\
8 a 10 & Risco alto & Necessária o quanto antes \\
11 & Risco muito alto & Necessária imediatamente \\
\hline
\end{tabular}

Fonte: Adaptado de Ergolândia 7.0.

O Diagrama de Corlett é um dos instrumentos utilizados para analisar os sintomas musculoesqueléticos em um contexto de saúde ergonômico, o que permite a identificação de sintomas por intermédio do trabalhador, assim como a necessidade de procura por recursos de saúde, assim como a interferência na realização das atividades (Figura 1).

A opção pelas três ferramentas de avaliação possibilita a obtenção de uma melhor interpretação dos resultados e a melhor constatação dos problemas osteomusculares relacionados ao trabalho.

Durante a realização dessas atividades, foram coletados dados para a avaliação postural, por meio de observações visuais dos trabalhadores, durante a execução das atividades, além de registros fotográficos e vídeos. Posteriormente, foi realizada a análise detalhada e a aplicação dos métodos.

Além disso, foram avaliadas as condições ambientais durante o período de condução dos experimentos e coletados os dados diários de temperatura e umidade relativa, de maneira que fossem observadas, também, as médias durante o período de trabalho. 


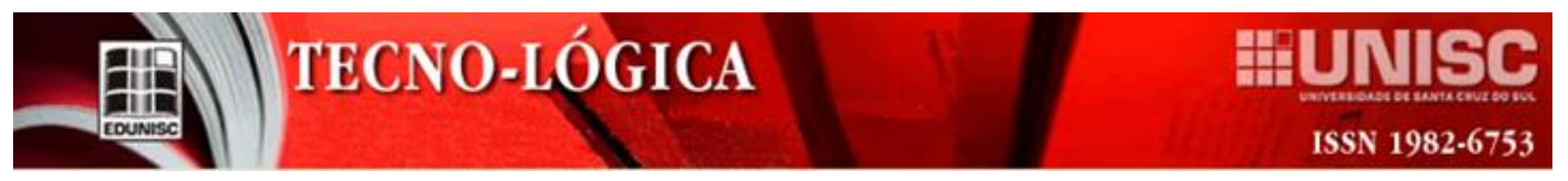

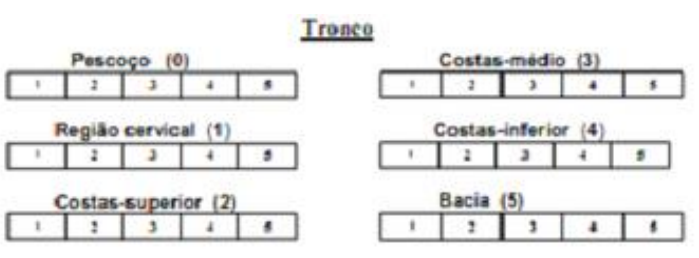

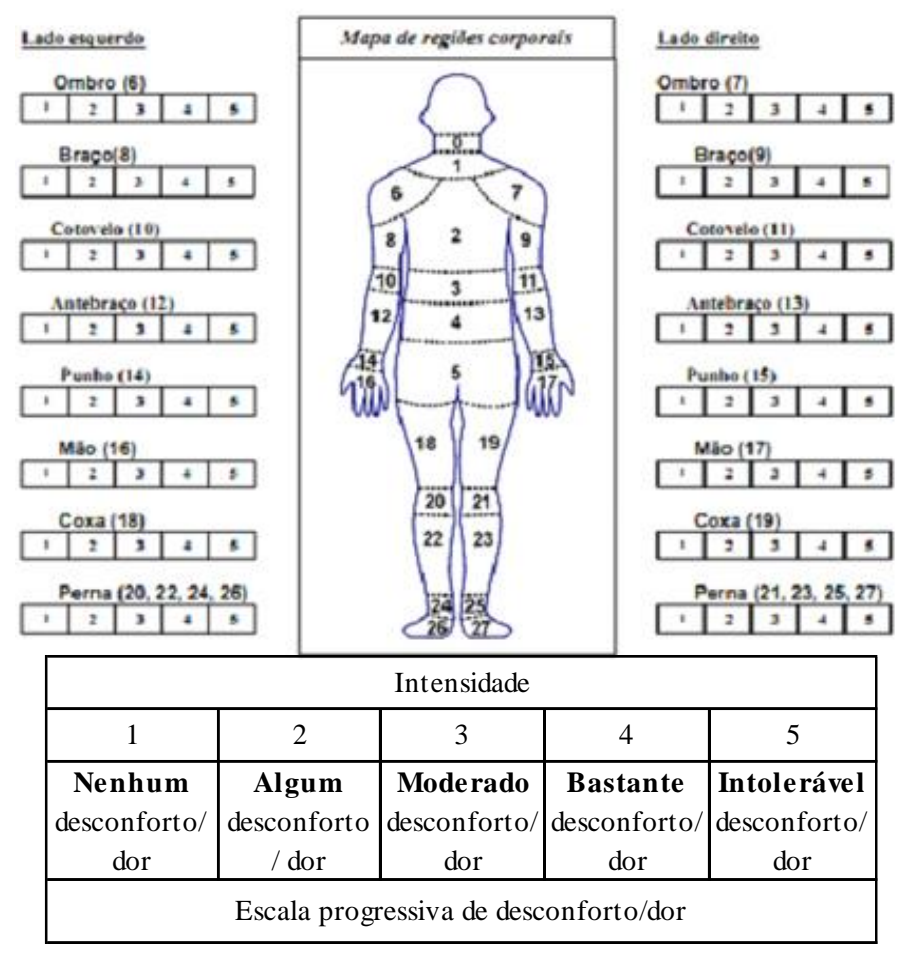

Figura 1. Diagrama de Corlett.

\section{Resultados e discussões}

A partir das observações das atividades realizadas no setor de floricultura, foi verificada a necessidade imediata de mudança postural nas atividades desenvolvidas, visto que os vasos estavam distribuídos diretamente no chão e os trabalhadores não tomavam nenhum cuidado com a postura durante a execução das atividades, nas quais era provocado o extremo desvio da coluna na execução e, como resultado, diversas dores (Figura 2).

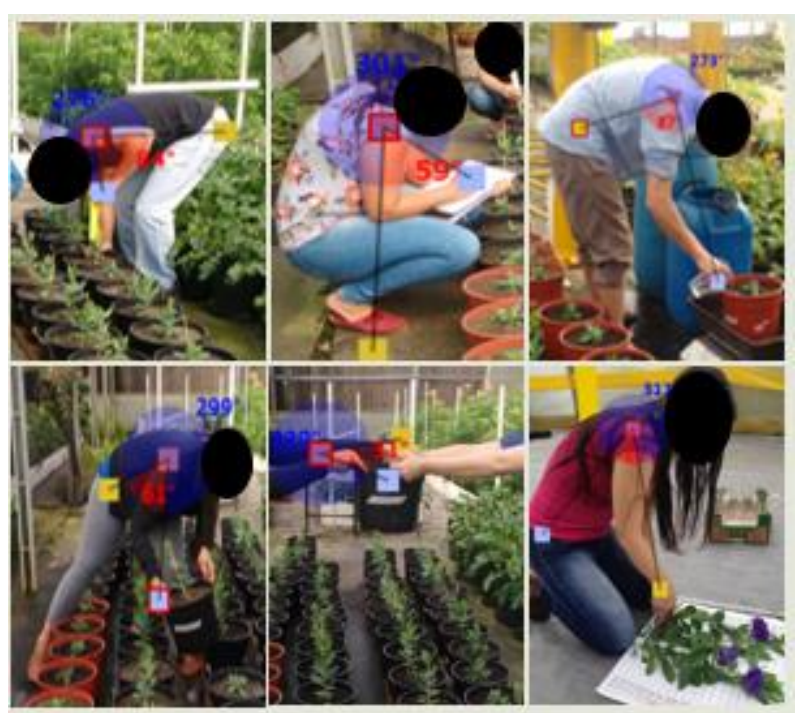

Figura 2. Posições comumente praticadas pelos trabalhadores analisados

Os principais fatores presentes nas atividades dos trabalhadores estudados e que desencadeiam as lesões e o desconforto estão ligados às posturas inadequadas, à velocidade da realização das atividades, à repetitividade, à duração e às condições do ambiente, o que corrobora com o estudo de Possebom et al. [6].

Por meio da análise realizada pela ferramenta de análise biomecânica RULA para as atividades desenvolvidas, são evidentes os problemas posturais encontrados, conforme os resultados obtidos na Tabela 3 .

Tabela 3. Resultados relativos à análise biomecânica RULA para as atividades desenvolvidas

\begin{tabular}{cccc} 
Atividade* $^{*}$ & Nível & Pontuação & Nível de intervenção \\
\hline 1 & 4 & 7 & $\begin{array}{c}\text { Devem ser introduzidas mudanças } \\
\text { imediatamente }\end{array}$ \\
\hline 2 & 3 & 6 & $\begin{array}{c}\text { Deve-se realizar uma } \\
\text { investigação. Devem ser } \\
\text { introduzidas mudanças }\end{array}$ \\
\hline 3 & 4 & 7 & $\begin{array}{c}\text { Devem ser introduzidas mudanças } \\
\text { imediatamente }\end{array}$ \\
\hline 4 & 4 & 7 & $\begin{array}{c}\text { Devem ser introduzidas mudanças } \\
\text { imediatamente }\end{array}$ \\
\hline 5 & 3 & 6 & $\begin{array}{c}\text { Deve-se realizar uma } \\
\text { investigação. Devem ser } \\
\text { introduzidas mudanças }\end{array}$ \\
\hline
\end{tabular}

*Onde as atividades são: 1- Avaliação de crescimento de plantas, 2- Preenchimento da planilha de dados in loco, 3- Realização de irrigação dos vasos por meio de pesagens, 4- Movimentação dos vasos e 5- Avaliação final das plantas 


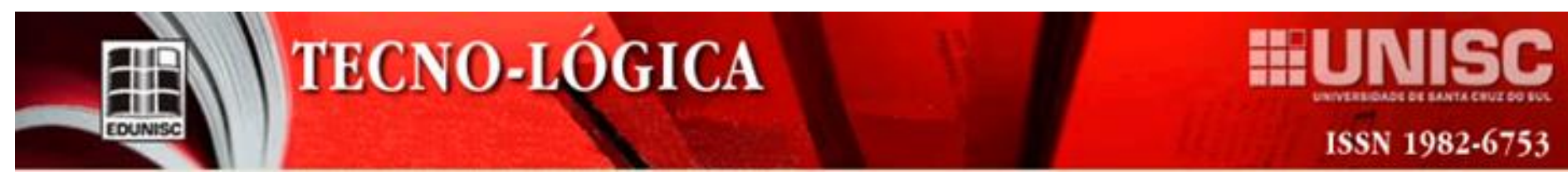

Segundo Cardoso Junior [12], a utilização deste método proporciona uma avaliação rápida em relação às cargas impostas ao sistema musculoesquelético dos trabalhadores, em função da postura e forças exercidas, sem a necessidade da utilização de equipamentos especiais.

De outro modo, pela utilização da análise realizada pela ferramenta biomecânica REBA, os resultados encontrados foram os seguintes (Tabela 4).

Tabela 4. Resultados relativos à análise biomecânica REBA para as atividades executadas

\begin{tabular}{cccc} 
Atividade* $^{*}$ & Pontuação & Significado & Nível de intervenção \\
\hline 1 & 9 & Risco Alto & Necessária o quanto antes \\
\hline 2 & 6 & $\begin{array}{c}\text { Risco } \\
\text { Médio }\end{array}$ & Necessária \\
\hline 3 & 7 & $\begin{array}{c}\text { Risco } \\
\text { Médio }\end{array}$ & Necessária \\
\hline 4 & 8 & Risco Alto & Necessária o quanto antes \\
\hline 5 & 9 & Risco Alto & Necessária o quanto antes \\
\hline
\end{tabular}

* Onde as atividades são: 1- Avaliação de crescimento de plantas, 2- Preenchimento da planilha de dados in loco, 3- Realização de irrigação dos vasos por meio de pesagens, 4- Movimentação dos vasos e 5- Avaliação final das plantas

Independentemente da metodologia de análise utilizada, foi verificado que todas as atividades avaliadas apresentaram a necessidade de intervenção imediata, a fim de evitar problemas futuros aos trabalhadores do setor.

Segundo Lopes e Oliveira [13], deve haver uma maior orientação dos trabalhadores sobre a manutenção da coluna de forma ereta e o flexionamento dos joelhos durante a realização das atividades. Essas medidas são necessárias, pois concentram os esforços nas pernas, o que evita a sobrecarga na coluna lombar.

Além dos resultados encontrados por meio dos métodos supracitados, a utilização do Diagrama de Corlett demonstrou o potencial aparecimento de fadigas musculares nos trabalhadores, relacionadas às posturas assumidas durante a execução das atividades. Em entrevista com três trabalhadores, foi verificado o surgimento de diversas dores em situações demasiadamente elevadas, tanto no final do turno de trabalho quanto nos dias posteriores, dependendo da intensidade de trabalho do dia, o que pode ser atestado na Figura 3.

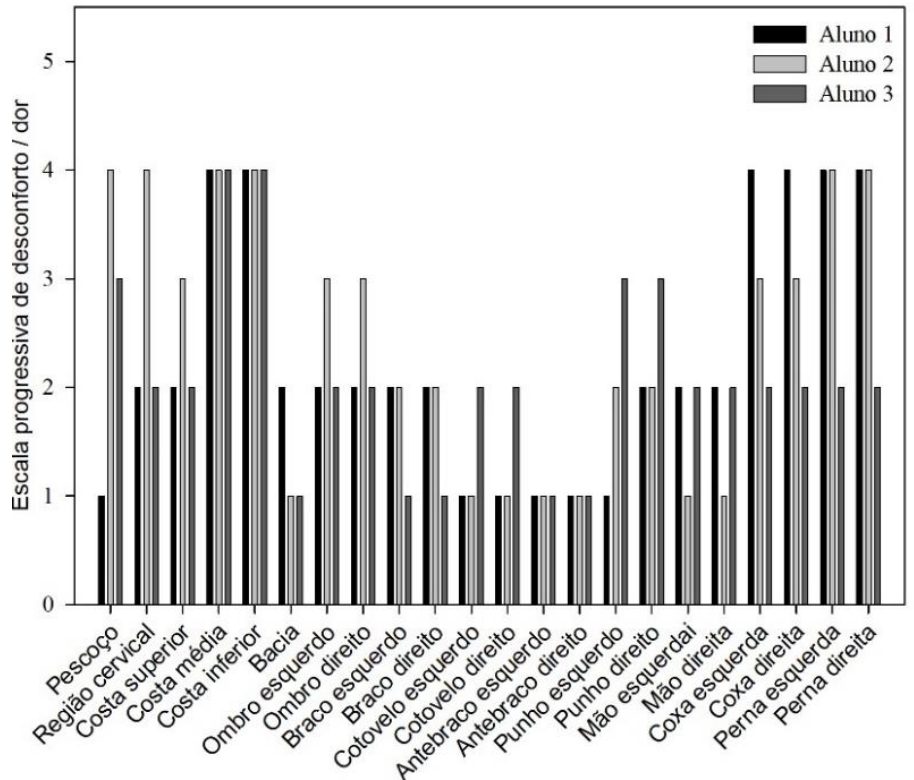

Figura 3. Principais desconfortos/dores que os trabalhadores apresentaram na execução das atividades desempenhadas

Segundo Vergara et al. [8], a ocorrência de fadigas musculares está ligada à sobrecarga muscular, que permanece localizada em um determinado músculo e que acarreta numa redução de desempenho do músculo fadigado, não apenas pela redução da força, mas também pela diminuição da velocidade dos movimentos. Os autores ainda referem que a falta de tratamento de fadigas musculares pode ocasionar em disfunções osteomusculares que, por sua vez, resultam no grande prejuízo à funcionalidade, além da produtividade do trabalhador. Como resultado, há problemas de coordenação e o aumento de erros e acidentes durante a execução das atividades.

O desenvolvimento de atividades em locais fora da zona de conforto muscular faz com que o trabalhador apresente desconforto e a perda da motivação para a realização do seu trabalho [6]. Assim, diminui a velocidade de reação para desempenhar as atividades, além da perda de precisão e, como resultado, o possível aumento de incidência de acidentes de trabalho [14].

Outro fator importante de ser salientado concerne a baixa consciência dos trabalhadores com relação à própria segurança, principalmente no que se refere à utilização de EPI's e outros equipamentos de segurança, pois, muitas vezes, os trabalhadores apresentam desconfortos ao utilizarem esses equipamentos.

A necessidade de considerar as variáveis ambientais durante a realização de trabalhos em locais fechados está relacionada com a importância que exercem, assim como as 
variáveis posturais, visto que um ambiente com condições climáticas desfavoráveis resulta em fadiga, no esgotamento físico e nervoso, na irritação, na redução do comprometimento e no rendimento, além da exposição do organismo a diversas doenças [15].

De acordo com as Normas Regulamentadoras do Ministério do Trabalho e Emprego- NR 15 [16], o limite de tolerância para exposição do trabalhador ao calor não deve ultrapassar os níveis de $25,26,7$ e $30^{\circ} \mathrm{C}$ para trabalhos classificados como leves, moderados e pesados, respectivamente. E de acordo com a NR 17 [17], a umidade relativa do ar não deve ser inferior a $40 \%$.

Dessa forma, durante a realização da análise nos postos de trabalho dos trabalhadores, foi verificada a temperatura média durante o dia de $24,3^{\circ} \mathrm{C}$ e a umidade relativa de $80,10 \%$. Esses resultados já eram esperados, tendo em vista o ambiente controlado para a produção de flores.

Segundo Couto [15], a execução de atividades com condições climáticas desfavoráveis ocasiona no aumento da desconcentração do trabalhador, à medida que pioram as condições de trabalho e, como resultado, a perda de eficiência e segurança no trabalho.

Portanto, em relação à análise das atividades, algumas intervenções podem ser sugeridas, tais como: a implantação de bancadas para alocação dos vasos, de maneira que seja reduzida a carga sobre a coluna e eliminada a necessidade dos trabalhadores se curvarem para levantar os vasos do chão; a proposição de uma mesa e cadeira, próximas ao local dos experimentos, a fim de facilitar os apontamentos necessários; a conscientização dos trabalhadores sobre a necessidade da utilização dos EPI's, como as botas e as luvas; quando houver a necessidade de carregar os vasos durante as atividades, optar por vasos com uma boa pega, visto que facilitam o transporte; implantação de bancadas de trabalho em conjunto com bancos de apoio adequados para os envolvidos nas atividades de análise experimental.

Por meio das adequações sugeridas, os trabalhadores podem desenvolver seu trabalho de maneira menos desgastante e debilitante, além de permanecerem motivados e felizes com a execução de suas atividades, assegurada a sua segurança e saúde.

\section{Conclusões}

O estudo concluiu que através da utilização de diferentes métodos de avaliação, o posto de trabalho não apresenta condições apropriadas para as atividades. Portanto, há a necessidade de investigação e a implantação de possíveis mudanças para corrigir os riscos à saúde dos trabalhadores.

A análise ergonômica do trabalho possibilitou verificar as situações que podem desencadear dor e desconforto nos trabalhadores. Todas as atividades analisadas não apresentaram conformidade quanto à postura empregada e, por isso, foi verificada a necessidade imediata de intervenção.

As principais dores estão concentradas na coluna e nos membros inferiores, em decorrência das posturas inadequadas, executadas durante as atividades. Assim, a utilização de bancadas para a realização dos trabalhos favorece a redução das cargas sobre a coluna e membros inferiores e elimina a necessidade de efetuar as tarefas com postura curvada.

\section{Agradecimentos} CAPES.

LASERG/UFSM, Setor de floricultura da UFSM e

\section{ASSESSMENT OF ERGONOMIC RISK IN ACTIVITY IN THE FLORICULTURE SECTOR}

ABSTRACT: In recent years, the floriculture sector has presented a high level of growth. However, there is a problem in this sector linked to workers' health care. This way, the aim of this study is to perform an ergonomic workplace analysis (EWA) applied to the working position of graduate students in the floriculture sector. This study was conducted in a protected environment, in the floriculture sector of the Colégio Politécnico of the Federal University of Santa Maria. In order to perform the ergonomic workplace analysis, the activities performed by the graduate students during scientific experiments were assessed. Thus, five activities were analyzed: plant growth evaluation, data sheet filling in loco, vase irrigation by weighing, transportation of vases and final plant evaluation. To perform the analyzes, data were collected for postural assessment by means of visual observations of the workers during the execution of activities, as well as photographic and video recordings. Subsequently, a detailed analysis was performed by applying the methods RULA, REBA, using the software Ergolândia 7.0 and the Corlett Diagram. This way, the ergonomic workplace analysis allowed to verify the situations that may trigger pain and discomfort in workers, as well as to evidence that the analyzed activities did not show compliance to the employed posture, requiring immediate intervention.

Keywords: Ergonomics. Protected environment. Rula. Reba. Corlett Diagram. 


\section{Referências}

[1] PESSA, S. L. R.; PEREIRA, K. S. M. Aplicação de Métodos Ergonômicos para Proposição de Melhorias ao Trabalho. Revista FSA, Vol. 15, n. 4, p 192-209, 2018

[2] WILDER, H. D.; ORJUELA, R.; ERLEY, M. Factores laborales y extralaborales de floricultores con Síndrome del Túnel del Carpo: cundinamarcaColombia 2013. Medicina y Seguridad del Trabajo, Vol. 62, n. 244, p. 199-211, 2016

[3] MARADEI-GARCÍA, M. F.; DELGADO-GAMBOA, A. C.; ESPINELCORREAL, F. Influencia de la postura durante el corte de flores en la fuerza de aprehensión. Revista de Salud Pública, Vol. 14, p. 460-469, 2012

[4] GERALDO, A. P. El Túnel Carpiano: Riesgo ergonómico en trabajadoras de cultivo de flores. Revista Ingeniería, Matemáticas y Ciencias de la Información, Vol. 1, n. 1, p 15-24, 2014

[5] CARLOTTO et al. Psicologia da saúde ocupacional: uma revisão integrativa. Aletheia Vol.50, n.1-2, p.143-153, 2017

[6] POSSEBOM et al. Avaliação ergonômica em um viveiro florestal de Santa Maria, RS. Tecno-Lógica, Vol. 21, n. 1, p. 30-36, 2017

[7] PINTO, C. C. A relação entre ergonomia e qualidade de vida no trabalho: uma revisão bibliográfica. Revista Ação Ergonômica, Vol. 13, n. 1, p. 96-112, 2019.

[8] VERGARA et al. Análise Ergonômica da atividade de Jardinagem e Paisagismo. Produção em Foco, Vol. 2, n. 1, p 85-105, 2012

[9] GOMEZ-GALAN et al. Musculoskeletal disorders: OWAS review. Industrial health, Vol. 55, n. 4, p. 314-337, 2017

[10] BOLICO DA SILVA, V. Análise e identificação dos riscos ergonômicos em atividades de modelagem do vestuário em estudantes. Revista Ação Ergonômica, Vol. 13, n. 1, p 71-95, 2019.

[11] FIEDLER et al. Avaliação da carga de trabalho físico exigido em operações de produção de mudas ornamentais no Distrito Federal: Estudo de Caso. Sociedade de Investigações Florestais. Revista Árvore, Vol. 31, n. 4, p 703-708, 2007

[12] TAKALA et al. Systematic evaluation of observational methods assessing biomechanical exposures at work. Scandinavian journal of work, environment \& health. Vol. 36, n 1, p. 3-24, 2010

[13] CARDOSO JUNIOR, M. M. Avaliação ergonômica: Revisão dos métodos para avaliação postural. Revista produção online, Vol. 6, n. 3, p 133-154, 2006.

[13] LOPES, E. S.; OLIVEIRA, F. M. Avaliação da carga de trabalho físico e da postura de trabalhadores na produção de mudas florestais. Cerne, Vol. 17, n. 4,p 573-582, 2011

[14] ALVES et al. Avaliação do ambiente de trabalho na propagação de Eucalyptus spp. Revista Brasileira de Engenharia Agrícola e Ambiental, v. 6, n. 3, p. 481-486, 2002

[15] COUTO, H. A. Ergonomia aplicada ao trabalho- conteúdo básico- guia prático. Belo Horizonte: Editora ERGO, 2007.

[16] NR15- Norma Regulamentadora: Atividades e condições insalubres. ABNTAssociação Brasileira de Normas Técnicas. Disponível em: <http://www.guiatrabalhista.com.br/legislacao/nr/nr15.htm>

[17] NR17- Norma Regulamentadora: Ergonomia. ABNT- Associação Brasileira de Normas Técnicas. Disponível em:<http://www.guiatrabalhista.com.br/legislacao/nr/nr17.htm> 